\title{
Did brightest cluster galaxies experience more than one star formation epoch?
}

\author{
D. N. Viljoen ${ }^{1}$ and S. I. Loubser ${ }^{1}$ \\ ${ }^{1}$ Centre for Space Research, North-West University, Potchefstroom, 2520, South Africa \\ email: 20569513@nwu.ac.za
}

\begin{abstract}
We use the full spectrum fitting ability of $U L y S S$, with the Pegase.HR stellar population model to fit the observed spectra of 40 brightest cluster galaxies in order to determine whether a single or a composite stellar population provided the most probable representation of the star formation history (SFH). We find that some galaxies in the sample have more complex SFHs.
\end{abstract}

Keywords. galaxies: evolution, galaxies: general - galaxies: stellar content.

\section{Introduction}

Recent literature, i.e. Loubser (2009) refer to brightest cluster galaxies (BCGs) as the central, dominant galaxy in a cluster with a typical mass of $\sim 10^{13} \mathrm{M}_{\odot}$ (Katayama et al. 2003). It has been widely excepted that BCGs are dormant elliptical galaxies with a red photometric color, implying that old stellar populations are present. However, Liu, Mao \& Meng (2012) have found that some BCGs have the presence of blue cores and UV excess which implies that star formation recently took place.

\section{Results and Conclusions}

We use the full spectrum fitting software package $U L y S S$ with the Pegase.HR stellar population model to fit this model against the observed spectra of 40 galaxies from Loubser (2009), to determine whether a simple or composite stellar population were a more probable representation of the star formation histories (SFHs). We found that 22 galaxies could be represented by a single stellar population (SSP) and the remaining 18 by composite stellar populations.

Our findings suggest that although $55 \%$ of the sample could be represented by an SSP, the remaining 18 galaxies experienced more than one star formation event. Hence, some BCGs have a more complex SFH than first assumed.

\section{Acknowledgments}

We thank the Square Kilometre Array project for the financial support.

\section{References}

Katayama, H., Hayashida, K. I., Takahara, F., \& Fujita, Y. 2003, ApJ, 585, 687

Liu, F. S., Mao, S., \& Meng, X. M. 2012, MNRAS, 423, 422

Loubser, S. I. 2009, Kinematics and stellar population in brightest cluster galaxies, Ph.D. thesis, University of Central Lancashire 